\title{
¿Qué estudiamos los ecólogos cuando investigamos los residuos plásticos en ambientes terrestres y dulceacuícolas de la Argentina?
}

\author{
A. Carolina Monmany-Garzia ${ }^{1, \mathbb{D} \text {; }}$ Agustina Malizia ${ }^{1 ;}$ Rocío Pazos ${ }^{2}$; Juliana M. \\ Ruiz Barrionuevo ${ }^{3}$; M. Fernanda Argarañáa; Nicolás Garello; ${ }^{5}$ Martín Blettleer; \\ Alberto Galindo-Cardona ${ }^{6,7}$; M. Angélica Occhionero ${ }^{3}$; Eduardo Martín ${ }^{3,6}$ \& \\ Ricardo E. De Cristobal ${ }^{8}$

\begin{abstract}
${ }^{1}$ Ambas autoras contribuyeron como primeras autoras. Instituto de Ecología Regional. Consejo Nacional de Investigaciones Científicas y Técnicas (CONICET) - Universidad Nacional de Tucumán (UNT). ${ }^{2}$ Instituto de Limnología "Dr. Raúl A.

Ringuelet", CONICET-Universidad Nacional de La Plata. ${ }^{3}$ Facultad de Ciencias Naturales e Instituto Miguel Lillo, UNT. ${ }^{4}$ Facultad de Bioquímica y Ciencias Biológicas, Universidad Nacional del Litoral. ${ }^{5}$ Instituto Nacional de Limnología, CONICET-Universidad Nacional del Litoral. ${ }^{6}$ Fundación Miguel Lillo. ${ }^{7}$ CONICET. ${ }^{8}$ Instituto de Química Biológica "Dr. Bernabé Bloj", Facultad de Bioquímica, Química y Farmacia, UNT, e Instituto Superior de Investigaciones Biológicas,
\end{abstract} CONICET-UNT.

\begin{abstract}
Resumen. La incógnita planteada en el título reunió a un grupo de investigadores en el simposio "Ecología de residuos plásticos: ¿Qué estudiamos en Argentina?", llevado a cabo en la XXVIII Reunión Argentina de Ecología. En esta comunicación hacemos un análisis del contexto nacional sobre residuos plásticos. Nuestros estudios en ecología de plásticos buscaron mitigar, por un lado, y cuantificar los residuos plásticos, por otro lado. Estos estudios ocurrieron en la mitad norte del país, en ambientes terrestres y acuáticos, y se enfocaron en diferentes materiales plásticos y en distintos organismos, aunque las bacterias fueron de interés para la mayoría de nosotros. Concluimos con preguntas guía para ecólogos, basadas en los Objetivos de Desarrollo Sostenible (ODS) de la ONU. Estas preguntas se relacionan con los ODS Agua Limpia y Saneamiento, Ciudades y Comunidades Sostenibles, Producción y Consumo Responsable y Vida Submarina y Sobre la Tierra.
\end{abstract}

[Palabras clave: ambientes acuáticos y terrestres, comunidades animales, contaminación plástica, Reunión Argentina de Ecología]

\begin{abstract}
Aвstract. What do we ecologists study when we investigate plastic waste in terrestrial and freshwater environments of Argentina? What kind of research questions do ecologists make in relation to plastic waste in Argentina? This question gathered a group of researchers at the symposium "Ecology of plastic waste: What do we study in Argentina?", held at the XXVIII Argentinian Ecology Meeting. In this communication we analyze the national context on plastic waste. Our studies on the ecology of plastics sought to mitigate, on the one hand, and to quantify plastic waste, on the other hand. These studies occurred in the northern half of the country, in terrestrial and aquatic environments, and were focused on different plastic materials and diverse organisms, though bacteria were of interest for the majority of us. We conclude with guiding questions for ecologists, based on the Sustainable Development Objectives of the UN. These questions are related to: Clean Water and Sanitation, Sustainable Cities and Communities, Responsible Consumption and Production, and Life Below Water and on Land.
\end{abstract}

[Keywords: animal communities, Argentinean Meeting of Ecology, plastic pollution, terrestrial and freshwater environments]

\section{INTRODUCCIÓN}

El plástico derivado del petróleo surgió en el siglo XX en los EE.UU., donde comenzó a producirse masivamente a partir de 1950 (American Chemical Society National Historic Chemical Landmarks 1993). Actualmente, la mayoría de los artículos que usamos está hecha de este material económico, ligero y duradero (Barnes et al. 2009), aunque su descarte excesivo causa acumulación en hábitats naturales, problemas para la fauna por ingestión o enredo, lixiviación de tóxicos

Editora asociada: María Semmartin 两 acmonmany@conicet.gov.ar asociados y transferencia de contaminantes a la fauna y a humanos (Thompson et al. 2009).

En el contexto de la XXVIII Reunión Argentina de Ecología en 2018, desarrollamos el simposio "Ecología de residuos plásticos: ¿Qué estudiamos en Argentina?", con los objetivos de presentar resultados sobre diferentes aspectos de la ecología de residuos plásticos en ambientes terrestres y dulceacuícolas, y promover la interacción entre los grupos dedicados al tema en la Argentina. Quisimos

Recibido: 28 de Marzo de 2019

Aceptado: 14 de Noviembre de 2019 
comprender la incidencia de los residuos plásticos en variados ambientes, bajo múltiples enfoques y a diferentes escalas. Debatimos sobre aspectos de transferencia e interacción con el público en general e identificamos puntos en común y vacíos de información para la temática en el país.

\section{Contexto actual}

El excesivo descarte de plásticos ha generado 11 "islas" de este material en los océanos (Eriksen et al. 2016), aunque el $80 \%$ proviene del continente (Eriksen et al. 2014). Las partículas plásticas difícilmente se degradan hasta convertirse en compuestos biológicamente reutilizables. Desde 1950 ingresan a los ecosistemas como macro- $(>5$ $\mathrm{mm})$ y micro- $(<5 \mathrm{~mm})$ plásticos, ya sea porque son ingeridas por organismos (Wilcox et al. 2015; Bessa et al. 2018), porque entran a los suelos junto al agua o a agroquímicos ( $\mathrm{Ng}$ et al. 2018), porque entran a las plantas por vía sistémica (Zorníková et al. 2011) o porque viajan por el aire como microfibras (Dris et al. 2017). Transitamos un proceso global con consecuencias tan extendidas en el tiempo que la contaminación plástica se ha propuesto como una de las huellas del Antropoceno (de Souza Machado et al. 2018; Malizia and Monmany-Garzia 2019).

Algunas cifras sugieren que los ecólogos estamos atrasados en el problema del plástico respecto de la importancia que tiene para la sociedad (Figura 1). Y aunque los estudios sobre ecología de residuos plásticos se incrementaron en la última década, se caracterizan por concentrarse en el hemisferio norte, en países industrializados (Barnes et al. 2009; Pérez et al. 2017; Blettler et al. 2018), en vertebrados (Wilcox et al. 2015; Lusher et al. 2017) y en ecosistemas marinos (Li et al. 2016; Malizia and Monmany-Garzia 2019). El alcance de la contaminación plástica y el interés general que despierta abre oportunidades únicas de investigación e impacto social para la investigación en ecología. Por ejemplo, al menos cinco de los recientes Objetivos de Desarrollo Sostenible (ODS) propuestos por las Naciones Unidas se relacionan con la contaminación plástica.

La disposición final de residuos sólidos urbanos (RSU) no está totalmente regulada por

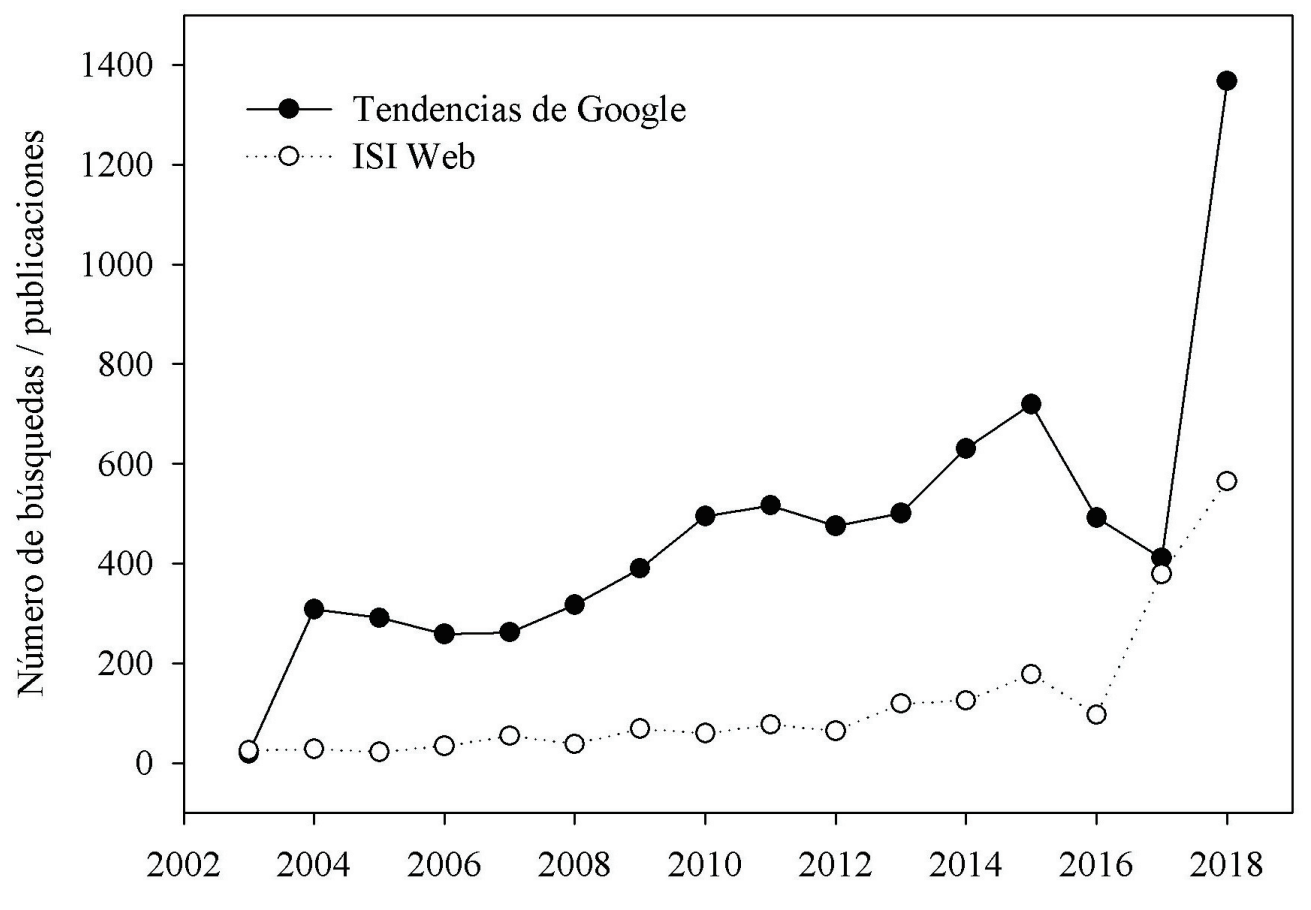

Años

Figura 1. Número total de búsquedas o publicaciones con el término "plastic pollution" (contaminación plástica) en Google Trends (línea sólida) y en ISI Web of Science (línea punteada), respectivamente, desde 2003 al 2018.

Figure 1. Total number of records or publications with the term "plastic pollution" in Google Trends (solid line) and in ISI Web of Science (discontinuous line), respectively, from 2003 to 2018. 
los Estados del mundo. Tampoco se respetan los límites políticos para su disposición, lo cual genera efectos ambientales y sociales negativos. En la Argentina, la población es altamente urbana $(90 \%)$ y genera en promedio más RSU comparado con Sudamérica: Argentina $=1.15 \mathrm{~kg} \cdot \mathrm{hab}^{-1} \cdot$ día $^{-1}$ vs. otros $=0.93$ kg.hab ${ }^{-1} \cdot$ día $^{-1}$ (Netwall et al. 2014). Aunque existe la Ley Nacional de Presupuestos Mínimos Ambientales No 25916/2004, que regula la gestión de estos residuos, y de que la mayor parte de las provincias tiene un plan de gestión integral (Pérez et al. 2017), aún hay ciudades que utilizan vertederos no regulados (Observatorio Nacional para la Gestión de los Residuos Sólidos Urbanos 2016). El 39\% de los argentinos no dispone sus residuos en rellenos sanitarios (Pérez et al. 2017), y las provincias del norte del país son las que más necesitan mejorar este aspecto. En este contexto presentamos los siguientes estudios ecológicos sobre residuos plásticos.
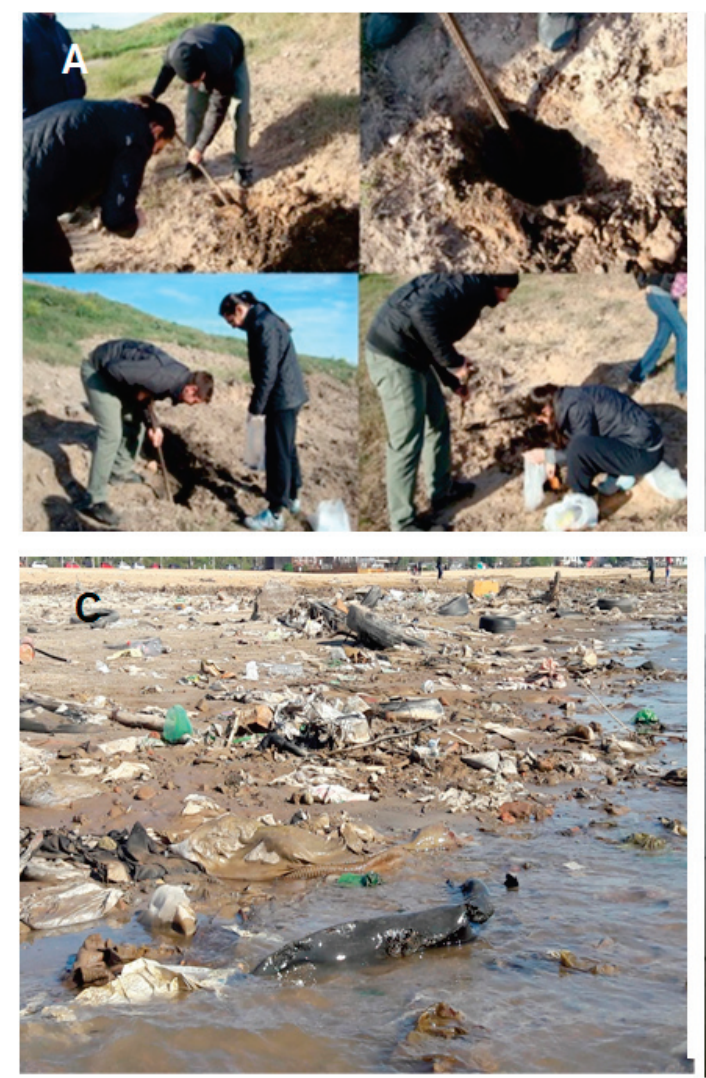

\section{DE ESTE A OESTE Y DE LO MICRO A LO MACRO}

En basurales de Santa Fe y del mundo, los plásticos conforman la mitad de los residuos. Un tipo común de residuo es el polipropileno biorientado (BOPP), que por su bajo costo y alta versatilidad se utiliza en muchos productos, incluyendo embalajes; por ello se acumula en la naturaleza. En su estudio sobre bacterias aisladas en suelos de rellenos sanitarios que degradan materiales plásticos, Argarañá y colaboradores (resultados no publicados) presentaron a la degradación microbiana como una posible tecnología de minimización de este tipo de residuos. Durante la biodegradación, diferentes enzimas de los microorganismos descomponen los polímeros complejos en moléculas más simples para ser utilizados como fuentes de carbono y energía. Para probar cómo actúan microorganismos aislados del relleno sanitario de Santa Fe (Figura 2A), el equipo aisló e identificó estos
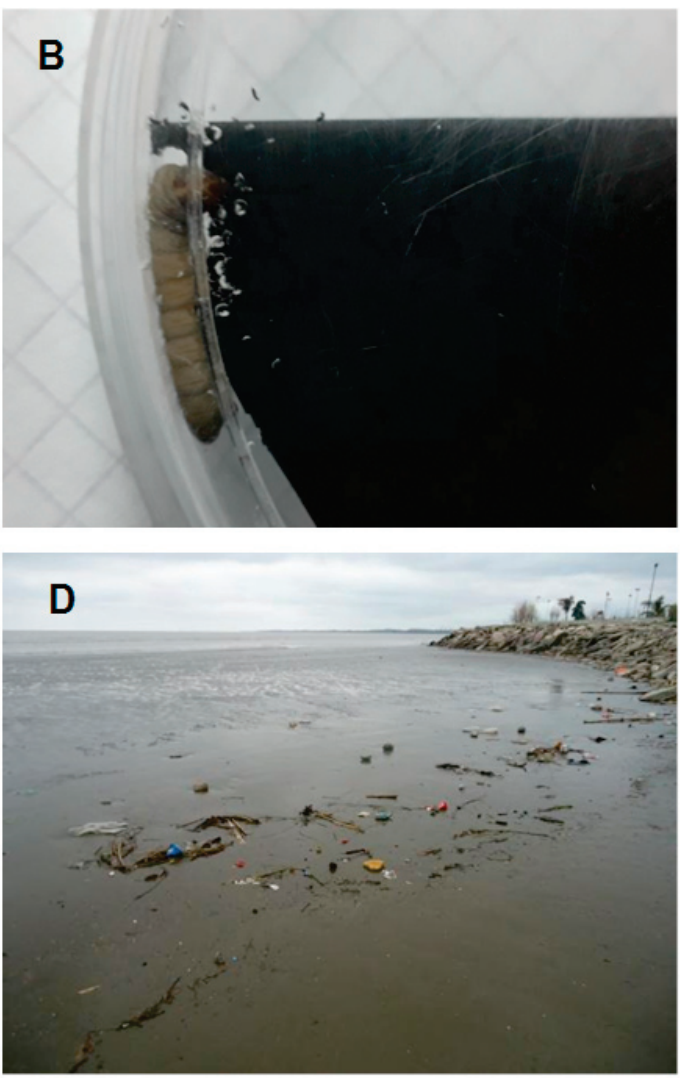

Figura 2. Imágenes representativas de los estudios presentados durante el simposio "Ecología de residuos plásticos: ¿Qué estudiamos en Argentina?", en la XXVIII Reunión Argentina de Ecología. A) Muestreos de bacterias en rellenos sanitarios de Santa Fe; B) Galleria mellonela consumiendo silobolsa en ensayos de laboratorio; C) Sitio de muestreo de biota y residuos plásticos en el río Paraná; D) Sitio de muestreo de biota y residuos plásticos en el Río de la Plata.

Figure 2. Images representing the studies presented during the symposium "Ecology of plastic waste: What do we study in Argentina?", at the XXVIII Argentinian Ecology Meeting. A) Bacteria samplings in landfills of Santa Fe; B) Galleria mellonela consuming silo-bags in lab assays; C) Biota and waste sampling site in the Paraná river; D) Biota and waste sampling site in the de la Plata river. 
microorganismos y desarrolló un método de screening para evaluar su potencial degradativo sobre el BOPP. Se determinaron tanto el nivel de degradación y los cambios estructurales y moleculares sufridos por el material expuesto a microorganismos seleccionados mediante microscopía electrónica de barrido y espectroscopía infrarroja con transformada de Fourier. En los aislamientos que mostraron mayor capacidad degradativa se detectó la presencia del gen alk B, que codifica para una de las enzimas postuladas como iniciadoras de este proceso. A corto plazo, los principales interrogantes plantean si es posible incrementar el potencial biodegradador de estas bacterias mediante la obtención de recombinantes, reduciendo los tiempos de degradación. A su vez, investigarán si es posible obtener mejores resultados utilizando consorcios bacterianos.

En un ecosistema productivo, Ruiz Barrionuevo y colaboradores (resultados no publicados) exploraron el consumo de plástico de silobolsas de dos especies de polillas, Achroia grisella F. y Galleria mellonella L. (Lepidoptera, Pyralidae). En Tucumán, registraron estas dos especies de polillas consumiendo silobolsas en colmenas abandonadas (Chalup et al. 2018). Para entender si la presencia de microorganismos sobre el cuerpo degradaba el plástico, primero determinaron si existían diferencias en el consumo de silobolsas entre larvas lavadas con antibióticos y controles sin antibiótico (Figura 2B). Las larvas se aislaron y monitorearon en cajas de Petri individuales conteniendo discos de silobolsas limpios y pesados, y el consumo se determinó a partir de la diferencia entre el peso inicial y el peso final de los discos. El consumo de silobolsa no reveló efectos derivados de la presencia de los microorganismos, y todas las larvas consumieron silobolsa hasta completar su desarrollo. Estos resultados preliminares sugieren que bacterias de la superficie corporal de las larvas no fueron agentes degradadores. Asimismo, aportan nuevos datos sobre alternativas de biodegradación (Bombelli et al. 2017). Actualmente, se aisló la microbiota del tracto digestivo de G. mellonella y se cultivaron cepas en distintos medios de cultivos para identificación. Además, se extrajo ADN genómico del tracto digestivo de larvas consumiendo cera y silobolsa para conocer el consorcio bacteriano. Finalmente, se identificó al parasitoide Apanteles galleriae (Hymenoptera, Braconidae) atacando a larvas de $A$. grisella consumiendo silobolsa
(Galindo-Cardona et al. 2019), lo cual mostró que interacciones ecológicas clave continuaron ocurriendo bajo una dieta de plástico.

En el contexto de los ecosistemas fluviales, Blettler y colaboradores (2017) estudiaron la contaminación plástica en el río Paraná (Figura 2C). Mediante un análisis bibliométrico identificaron faltantes, inconsistencias y sesgos en el conocimiento de la contaminación plástica en ambientes dulceacuícolas del mundo, y en estudios a campo estimaron la distribución espacial y describieron los tipos de plásticos en sedimentos marginales y planicie del Río Paraná. Concluyeron que es necesario incrementar los estudios de campo para estimar la entrada total de plásticos desde los ríos hacia el mar. Asimismo, concluyeron que los microplásticos están mucho más estudiados que los macroplásticos, aunque el conocimiento del impacto de los microplásticos sobre la fauna dulceacuícola es muy limitado y el de los macroplásticos es prácticamente nulo. Pese a que muchos de los ríos más contaminados se encuentran en Asia, sólo el 14\% de los estudios totales se llevó a cabo en este continente, y resulta imprescindible mejorar el conocimiento de los países en desarrollo y con gestión de residuos deficiente. El estudio a campo sobre las márgenes del Río Paraná (Blettler et al. 2017) documentó altas cantidades de macroplásticos, en particular polietileno de alta y baja densidad, polipropileno, y poliestireno, compacto y expandido. Se hallaron más de 5200 partículas de microplásticos por $\mathrm{m}^{2}$, y la mayor abundancia se detectó aguas abajo de la ciudad de Paraná. Finalmente, el estudio reveló microplásticos en el tracto digestivo del 100\% de los peces estudiados. Un análisis comparativo con otros estudios del mundo demuestra que la contaminación plástica es un grave problema en el Río Paraná.

En el extremo oriental del país, Pazos y colaboradores estudiaron la abundancia de microplásticos en el agua, la biota y el sedimento de la costa del Río de la Plata (Figura 2D). Los microplásticos son considerados contaminantes emergentes que por escorrentía desde zonas urbanas (e.g., desagües) y desde los arroyos que las atraviesan pueden ser transportados a ambientes costeros, como los estuarios, y a ambientes marinos. Los estuarios son ecosistemas muy diversos, y el del Río de la Plata provee bienes y servicios como agua para consumo con fines recreacionales y para cría de peces de interés comercial. Sin 
embargo, también recibe escorrentía de la agricultura, descargas industriales y efluentes cloacales a lo largo de la costa argentina, lo cual genera el ingreso de contaminantes como los macroplásticos y microplásticos. El estudio reveló que todos los peces analizados contenían microplásticos (principalmente fibras) y que la cantidad de microplásticos por individuo fue más abundante en un sitio relacionado con una descarga cloacal. A su vez, la cantidad de microplásticos no varió significativamente entre peces con distintos hábitos alimentarios (Pazos et al. 2017). Por otra parte, el estudio realizado por Pazos et al. (2018) reveló la presencia de microplásticos en la columna de agua, en 10 sitios muestreados en la costa del río, entre San Isidro y Punta Indio, si bien la distribución fue irregular, con los valores máximos en la cercanía de las ciudades de Buenos Aires y de La Plata, seguido de las descargas cloacales de estas ciudades y del sitio ubicado en el frente de máxima turbidez del estuario. Comparando los rangos de tamaño entre organismos planctónicos y microplásticos en las muestras, fue evidente que la frecuencia de tamaños de una fracción de microplásticos coincidió con la frecuencia de tamaños más comunes de plancton y las formas fueron también similares, lo cual aumentaría la posibilidad de ingesta por organismos de distintos niveles tróficos, incluido el zooplancton. Por otro lado, la distribución de microplásticos en el sedimento intermareal también fue variable y los mayores registros fueron cercanos a La Plata. Los microplásticos aún no se encuentran regulados y necesitamos realizar más estudios para conocer el riesgo ambiental y sanitario que implica su presencia y abundancia. Actualmente, el equipo está estudiando la presencia de microplásticos en mejillones de la costa del río y analizando la colonización por parte de bacterias indicadoras de contaminación fecal en microplásticos del sedimento intermareal del río.

En resumen, los estudios ecológicos sobre residuos plásticos en la Argentina se llevan a cabo en unas pocas regiones y ecosistemas, y se enfocan en diferentes materiales plásticos, aunque también encontramos similitudes en los organismos bajo estudio y en la manera de abordar las preguntas (Tabla 1). Todos consideran el estudio a corto plazo de microorganismos en relación con el plástico. Esto se debe a que los microorganismos tienen ciclos de vida cortos y muestran una rápida respuesta ecológica; también son diversos en cuanto a las interacciones en las que se involucran, lo que genera resultados no siempre predecibles. Los estudios de Argarañá et al. y de Ruiz et al. (resultados no publicados) buscaron mitigar la contaminación y se concentran en ecosistemas terrestres, mientras que los de Bettler et al. (2017) y Pazos et al. (2017; 2018) buscaron cuantificar la contaminación y se concentraron en ecosistemas acuáticos. Algunos vacíos son evidentes en la compilación de nuestros esfuerzos. Por un lado, los estudios aquí mencionados se desarrollaron en el centro y norte del país por lo que necesitamos afianzar el estado de conocimiento en el sur. Además, la gama de materiales plásticos que ingresan a los ecosistemas naturales es mucho más amplia que la que examinaron nuestros estudios y necesitamos incorporar la trazabilidad (e.g., monitoreos espaciales y temporales) e interacciones ecológicas de diferentes tipos de desechos plásticos.

Tabla 1. Provincias, ambientes, organismos, tipos de plásticos estudiados y enfoques utilizados por los autores de este trabajo, según lo presentado en el simposio de la Reunión Argentina de Ecología 2018.

Table 1. Provinces, environments, organisms, plastic types studied, and approaches used by the authors of this study, according to the results presented at the Ecology Argentinean Meeting in 2018.

\begin{tabular}{lllll}
\hline Provincia & Ambiente & Organismo & Tipo de plástico & Enfoque \\
\hline Santa Fe & Basurales & Bacterias (genes) & $\begin{array}{l}\text { Polipropileno biorientado } \\
\text { (BOPP) }\end{array}$ & Biodegradación (mitigación)
\end{tabular}
$\begin{array}{ccc}\text { Tucumán Campos / agro Insectos (bacterias) } & \begin{array}{l}\text { Triple capa de polietileno de } \\ \text { baja densidad (silobolsas, SB) }\end{array}\end{array}$

Biodegradación (mitigación) 


\section{Potenciales INVESTIGACIONES Y COLABORACIONES PARA ECÓLOGOS}

Sugerimos utilizar los siguientes ODS de la ONU como guía para preguntas ecológicas a diferentes escalas espacio-temporales e incluyendo a profesionales de otras disciplinas.

\section{ODS 6 (Agua Limpia y Saneamiento)}

- En nuestro país, ¿Cuáles son las cuencas y la biota más afectadas por la contaminación plástica?

- ¿Cuánto se mueven los residuos plásticos (e.g., BOPP) a través del paisaje? ¿Cuántos quedan acumulados en los fondos de ríos y en otros cuerpos de agua? ¿Existe contaminación de microplásticos en napas freáticas?

- ¿Cuántos de los residuos plásticos desechados tierra adentro, hacia el oeste de la Argentina, contribuyen de manera efectiva a las islas marinas de basura plásticas?

\section{ODS 11 (Ciudades y Comunidades Sostenibles)}

- ¿Cuál es la percepción de los ciudadanos sobre el origen, el uso y la disposición final de los residuos plásticos?

- ¿Cómo se mide y cuál es el éxito de las campañas de concientización del uso responsable de los residuos plásticos en cada ciudad?

- ¿Cuánto residuo plástico se genera por día en casas, escuelas y lugares de trabajo? ¿En qué medida se relaciona esto con los ingresos económicos, con la ubicación geográfica y con otras variables socio-ecológicas? ¿Cómo contribuye la separación de residuos a su reducción?

\section{ODS 12 (Producción y Consumo Responsable)}

- ¿Cuántos tipos de residuos plásticos se generan en los distintos sistemas productivos de la Argentina, como la agricultura, la ganadería, etc.? ¿Cuál es el destino de los plásticos desechados en cada caso y cómo impactan en los servicios ecosistémicos que brinda la biota asociada?

- ¿Cuántos de los residuos destinados a reciclarse entran verdaderamente en las cadenas de reciclaje? ¿Cuáles son los impactos respecto a este manejo en los ecosistemas de la Argentina?

\section{ODS 14 (Vida Submarina)}

- ¿Cuál es la distribución y abundancia de los residuos plásticos en los ambientes marinos costeros del país? ¿Cuáles son las consecuencias para los ecosistemas submarinos?

- ¿Cómo es la degradación del plástico en el ambiente marino? ¿Cómo afecta esta degradación a las condiciones biológicas y fisicoquímicas de los océanos?

\section{ODS 15 (Vida Sobre la Tierra)}

- ¿En qué grado afecta la contaminación plástica a los distintos niveles tróficos? ¿Existen diferencias respecto a esta contaminación en las redes de ecosistemas naturales y en aquellas de ecosistemas productivos?

- ¿Cuál es la dinámica de los residuos plásticos en ambientes terrestres? ¿Cómo cambian las relaciones ecológicas del plástico en el tiempo y el espacio?

- ¿Cómo se relaciona la dinámica de los residuos plásticos con el cambio climático?

- ¿En qué grado cambia el comportamiento de los organismos por la contaminación plástica?

\section{CONCLUSIONES}

En la Argentina, los estudios con un enfoque ecológico acerca de los residuos plásticos son escasos. Los que reunimos en este trabajo, por un lado, buscan soluciones a la contaminación plástica en sistemas terrestres $y$, por otro, cuantifican la contaminación plástica en sistemas acuáticos. Los ecosistemas del sur del país deberían recibir más atención y es necesario una mayor comprensión de la incidencia dematerialesplásticosnoestudiados aún. Las bacterias fueron organismos de interés en todas las investigaciones reunidas, aunque los ecólogos deberíamos considerar la posibilidad de que la contaminación plástica alcance, eventualmente, a todos los organismos, interactuando e impactando en procesos y patrones. En este sentido, se plantea el desafío de optimizar los esfuerzos entre estudios descriptivos, experimentales y de co-diseño que respondan a preguntas locales urgentes y concretas.

Agradecimientos. Agradecemos a los organizadores de la XXVIII Reunión Argentina de Ecología por la oportunidad de organizar este simposio, por su apoyo logístico durante 
el evento y el apoyo económico para la participación de R. Pazos. La Secretaría de Estado de Innovación y Desarrollo Tecnológico de Tucumán financió la participación de A. C.
Monmany-Garzia en el simposio. Al público y colegas por sus preguntas y comentarios. También agradecemos los aportes de tres revisores anónimos al manuscrito final.

\section{REFERENCIAS}

American Chemical Society National Historic Chemical Landmarks. 1993. Bakelite: The World's First Synthetic Plastic. URL: https://tinyurl.com/y9fmwpjl.

Barnes, D. K. A., F. Galgani, R. C. Thompson, and M. Barlaz. 2009. Accumulation and fragmentation of plastic debris in global environments. Philosophical Transactions of the Royal Society B: Biological Sciences 364:1985-1998. https: //doi.org/10.1098/rstb.2008.0205.

Bessa, F., P. Barría, J. M. Neto, J. P. G. L. Frias, V. Otero, P. Sobral, and J. C. Marques. 2018. Occurrence of microplastics in commercial fish from a natural estuarine environment. Marine Pollution Bulletin 128:575-584. https://doi.org/ 10.1016/j.marpolbul.2018.01.044.

Blettler, M. C. M., E. Abrial, F. R. Khan, N. Sivri, and L. A. Espínola. 2018. Freshwater plastic pollution: Recognizing research biases and identifying knowledge gaps. Water Research 143:416-424. https://doi.org/10.1016/j.watres.2018.06.015.

Blettler, M. C. M., M. A. Ulla, A. P. Rabuffetti, and N. Garello. 2017. Plastic pollution in freshwater ecosystems: macro, meso-, and microplastic debris in a floodplain lake. Environmental Monitoring and Assessment 189:581. https: //doi.org/10.1007/s10661-017-6305-8.

Bombelli, P., C. J. Howe, and F. Bertocchini. 2017. Polyethylene bio-degradation by caterpillars of the wax moth Galleria mellonella. Current Biology 27:R292-R293. https://doi.org/10.1016/j.cub.2017.02.060.

Chalup, A., M. M. Ayup, A. C. Monmany Garzia, A. Malizia, E. Martin, R. De Cristóbal, and A. Galindo-Cardona. 2018. First report of the lesser wax moth Achroia grisella F. (Lepidoptera: Pyralidae) consuming polyethylene (silo-bag) in northwestern Argentina. Journal of Apicultural Research 57:569-571. https://doi.org/10.1080/ 00218839.2018.1484614.

Dris, R., J. Gasperi, C. Mirande, C. Mandin, M. Guerrouache, V. Langlois, and B. Tassin. 2017. A first overview of textile fibers, including microplastics, in indoor and outdoor environments. Environmental Pollution 221:453-458. https: //doi.org/10.1016/j.envpol.2016.12.013.

Eriksen, M., L. C. M. Lebreton, H. S. Carson, M. Thiel, C. J. Moore, J. C. Borerro, F. Galgani, P. G. Ryan, and J. Reisser. 2014. Plastic Pollution in the World's Oceans: More than 5 Trillion Plastic Pieces Weighing over 250,000 Tons Afloat at Sea. PLoS ONE 9:1-15. https://doi.org/10.1371/journal.pone.0111913.

Eriksen, M., M. Thiel, and L. Lebreton. 2016. Nature of plastic marine pollution in the subtropical gyres. Pp 135162 en H. Takada and H. K. Karapanagioti (eds.). Hazardous Chemicals Associated with Plastics in the Marine Environment. The Handbook of Environmental Chemistry. Springer International Publishing, Cham. Vol 78. https: //doi.org/10.1007/698_2016_123.

Galindo-Cardona, A., J. D. Achar, G. González-Brizuela, E. Martín, S. A. Salvo, and A. C. Monmany-Garzia. 2019. First report and molecular determination of Apanteles galleriae Wilkinson (Hymenoptera, Braconidae), a parasitoid of the lesser wax moth Achroia grisella F. (Lepidoptera, Pyralidae) in Northwest Argentina. Journal of Apicultural Research 58:550-552. https://doi.org/10.1080/00218839.2019.1626622.

Li, W. C., H. F. Tse, and L. Fok. 2016. Plastic waste in the marine environment: A review of sources, occurrence and effects. Science of the Total Environment 566-567:333-349. https://doi.org/10.1016/j.scitotenv.2016.05.084.

Lusher, A., P. Hollman, and J. Mendoza-Hill. 2017. Microplastics in fisheries and aquaculture: status of knowledge on their occurrence and implications for aquatic organisms and food safety. FAO Fisheries and Aquaculture Technical Paper. Food and Agriculture Organization of the United Nations, Rome, Vol 615. Pp. 147.

Malizia, A., and A. C. Monmany-Garzia. 2019. Terrestrial ecologists should stop ignoring plastic pollution in the Anthropocene time. Science of The Total Environment 668:1025-1029. https://doi.org/10.1016/ j.scitotenv.2019.03.044.

Netwall, A., C. Nuques, O. Garzonio, W. Vega, M. Soulier, C. Chantrill, A. Palacios, J. Cayo, I. Vinocur, G. Velázquez, K. Machado, and S. Campos. 2014. Propuesta Argentina para Préstamo Banco Interamericano de Desarrollo. ARL1151. Secretaría de Ambiente y Desarrollo Sustentable de la Nación y Ministerio de Turismo de la Nación Argentina, Buenos Aires, Argentina.

Ng, E. L., E. Huerta Lwanga, S. M. Eldridge, P. Johnston, H. W. Hu, V. Geissen, and D. Chen. 2018. An overview of microplastic and nanoplastic pollution in agroecosystems. Science of the Total Environment 627:1377-1388. https: //doi.org/10.1016/j.scitotenv.2018.01.341.

Observatorio Nacional para la Gestión de RSU. 2016. Mapas críticos nacionales en gestión de residuos urbanos. Ministerio de Ambiente y Desarrollo Sustentable, Buenos Aires, Argentina. URL: https://tinyurl.com/uk3brq9.

Pazos, R. S., D. E. Bauer, and N. Gómez. 2018. Microplastics integrating the coastal planktonic community in the inner zone of the Río de la Plata estuary (South America). Environmental Pollution 243:134-142. https://doi.org/10.1016/ j.envpol.2018.08.064.

Pazos, R. S., T. Maiztegui, D. C. Colautti, A. H. Paracampo, and N. Gómez. 2017. Microplastics in gut contents of coastal freshwater fish from Río de la Plata estuary. Marine Pollution Bulletin 122:85-90. https://doi.org/10.1016/ j.marpolbul.2017.06.007.

Pérez, N., M. Guagliano, A. Sánchez Rico, A. Lugones, A. Alvarez, R. Lomurno, A. Cabral, J. M. Millet, M. Rosso, 
N. Dobler, M. Vello, and A. Nicosia. 2017. Estudio de vigilancia tecnológica e inteligencia competitiva en ciencia, tecnología e innovación en residuos sólidos urbanos. Ministerio de Ciencia, Tecnología e Innovación Productiva de Argentina, Buenos Aires. Pp. 170.

de Souza Machado, A. A., W. Kloas, C. Zarfl, S. Hempel, and M. C. Rillig. 2018. Microplastics as an emerging threat to terrestrial ecosystems. Global Change Biology 24:1405-1416. https://doi.org/10.1111/gcb.14020.

Thompson, R. C., C. J. Moore, F. S. vom Saal, and S. H. Swan. 2009. Plastics, the environment and human health: current consensus and future trends. Philosophical Transactions of the Royal Society B: Biological Sciences 364:2153-2166. https://doi.org/10.1098/rstb.2009.0053.

Wilcox, C., E. Van Sebille, and B. D. Hardesty. 2015. Threat of plastic pollution to seabirds is global, pervasive, and increasing. Proceedings of the National Academy of Sciences 112:11899-11904. https://doi.org/10.1073/ pnas.1502108112.

Zorníková, G., A. Jarošová, and L. Hřivna. 2011. Distribution of phthalic acid esters in agricultural plants and soil. Acta Universitatis Agriculturae et Silviculturae Mendelianae Brunensis 59:233-237. https://doi.org/10.11118/ actaun201159030233. 